\title{
473165 - ELECTROCORTICOGRAPHIC (ECOG) ACTIVATION BY ANESTHETIC AGENTS DURING EPILEPSY SURGERY UNDER GENERAL ANESTHESIA - EVIDENCE BASED REVIEW
}

\author{
Sanjib Adhikary, M.D., Lakshmikumar Venkat Raghavan, MD FRCA, Pirjo \\ Manninen, MD FRCPC \\ Anesthesia, Toronto Western Hospital, Toronto, ON, Canada
}

Introduction: Intraoperative activation of epileptic foci and the intraictal spikes using electrocorticograph(ECOG) is important to determine the extent of resection and thus outcome of the epilepsy surgery. Various anesthetic agents have been used for this purpose.(1) The purpose of this study is to conduct a systematic review of the existing evidence in the literature related to ECOG activation by the anesthetic agents during epilepsy surgery under general anesthesia.

Methods: A literature search was performed combining the following terms: epilepsy surgery, electrocorticography, intraoperative, anesthesia, seizure activation in Medline, EMBASE, Cochrane (EBMR) and PsycInfo databases (1966-Nov 2007, English abstracts only, limited to human) using the Ovid search interface to identify and review studies related to ECOG activation by the anesthetic agents under general anesthesia. Studies involving awake patients and studies where scalp EEG was used for evaluation of epileptic activity were excluded. All randomized and non randomized trials were included in the study.

Results: Twenty-eight out of 55 articles reviewed met the inclusion criteria. The various agents used for activation included intravenous induction agents (3), opioid analgesics (10) and inhalational agents (15). The summaries of results are shown in Table 1. Among all the agents, opioid analgesics have been most extensively studied using controlled trials. Alfentanil and remifentanil have shown to be most effective in ECOG activation. Among inhalational agents sevoflurane has been extensively studied but it is less specific and the activation potential varies with the concentration. Non-opioid agents like methohexital have gone out of use in recent years and other intravenous induction agents have not been studied extensively in this regard. It was not possible to analyze the data using metananlysis, as the nature of the studies was totally inconsistent with respect to the variables.

Discussion: Most of the agents used for ECOG activation are based on institutional practice rather than evidence based. Among existing studies, short acting opioids demonstrate most specificity to the activation process. More studies are needed before a definite clinical guideline or protocol can be developed.

References: 1. Ragazzo PC et al. Brain Res Brain Res Rev. 2000 Apr; 32(1):316-27. 


\begin{tabular}{|c|c|c|c|c|c|}
\hline \multicolumn{5}{|c|}{ प्र०००343 } & \multirow[b]{7}{*}{3} \\
\hline 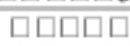 & 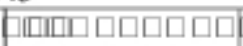 & IIIप口म & ПIIII $\square \square \square$ & 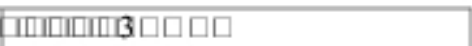 & \\
\hline 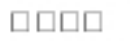 & 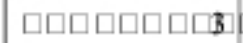 & पाшाшा & 팾 & 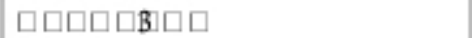 & \\
\hline ㅁㅁ & 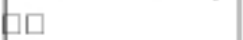 & & ㅁㅁㅁ & पि口०3 & \\
\hline & & & प्रणमा & पि口3 & \\
\hline & & & प्पि३म & $\mathrm{B}$ & \\
\hline 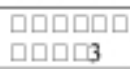 & $\mathrm{E} \square \square 4 \mathrm{AA} \square \square 3 \square \% \square$ & $\% 3 \quad \mu 3$ & पित & 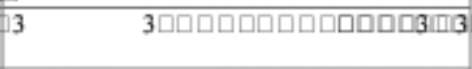 & \\
\hline प्र००स & 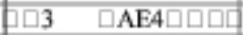 & $8 \square \square 8 \square \% \square \%$ & 6343 & 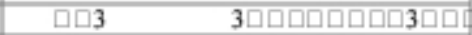 & की००3 \\
\hline परमपणि & 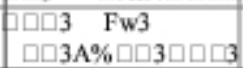 & $\mu^{3}$ & 003 & 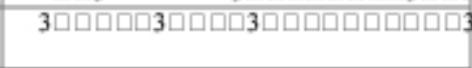 & 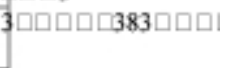 \\
\hline 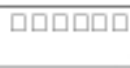 & 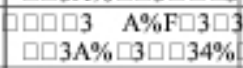 & $\begin{array}{l}34 \% \square w 33 \\
\square \square \square \square 3\end{array}$ & प्र०3 & 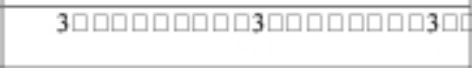 & 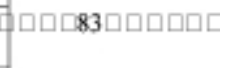 \\
\hline 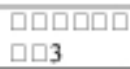 & 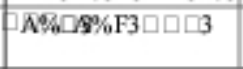 & $\square 3$ & पि口3 & 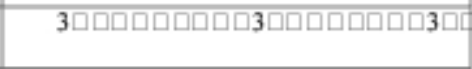 & पि口प3 \\
\hline 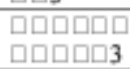 & $3 \mathrm{~A} 3 \square \square 3 \square \mathrm{Aw} 33$ & $\mu 3$ & $\square \square 3$ & 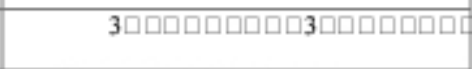 & 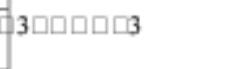 \\
\hline प्रमपर & $\begin{array}{l}3 \square \quad 4 \square \% \square 3 \\
\square \square \square 8 \square \square 3 \square \% \square \% 3\end{array}$ & $3 F 3 \mu \square \% \square 4$ & $43 \square \square \square 3$ & 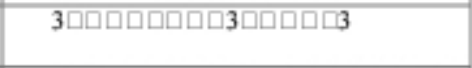 & \\
\hline प्रमपर & $\begin{array}{l}\text { पि口๐3 } 43 \square \square 3 \mathrm{~F} \\
\square \% \square \% 3\end{array}$ & 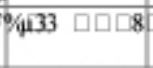 & का 303 & 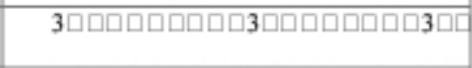 & 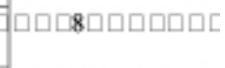 \\
\hline प्रिप्र & $\begin{array}{l}\text { पि口प3 FA3 } \square \square 3 \\
\square \% \square \% 3\end{array}$ & 4AßА $\square \square 8$ & 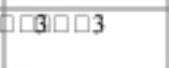 & 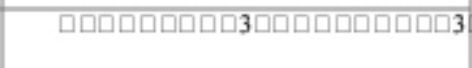 & 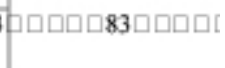 \\
\hline
\end{tabular}

